\title{
ANÁLISE DA SATURAÇÃO DE MgO PARA ESCÓRIAS DO SISTEMA CaO-SiO${ }_{2}-\mathrm{FeO}-\mathrm{MgO}-\mathrm{Al}_{2} \mathrm{O}_{3}{ }^{*}$
}

\author{
Rodolfo Arnaldo Montecinos de Almeida \\ Deisi Vieira ${ }^{2}$ \\ Wagner Viana Bielefeldt $t^{3}$ \\ Antônio Cezar Faria Vilela ${ }^{4}$
}

Resumo

As escórias de forno elétrico a arco (FEA) têm diversas funções, entre elas a melhoria da eficiência energética através do fenômeno da espumação. Por isso o estudo das escórias é de suma importância nos FEA's. Um dos fatores que determina a qualidade da espumação e a sua eficácia é presença de partículas sólidas na escória. A saturação de $\mathrm{MgO}$ é extremamente importante, pois além de garantir partículas sólidas garante também a compatibilidade química para minimizar o consumo do refratário. Neste trabalho, foi feito o estudo da saturação de $\mathrm{MgO}$ para escórias no sistema $\mathrm{CaO}-\mathrm{SiO}_{2}-\mathrm{FeO}-\mathrm{MgO}-\mathrm{Al}_{2} \mathrm{O}_{3}$, onde avaliou-se a influência que a basicidade binária, os teores de $\mathrm{FeO}$ e do $\mathrm{Al}_{2} \mathrm{O}_{3}$ tem sobre o mesmo. Foram feitos diagramas ternários, onde viu-se as diferenças que os teores de $\mathrm{Al}_{2} \mathrm{O}_{3}$ tem sobre os campos líquidos e as demais fases presentes. Foram feitos os diagramas de saturação isotérmicos (ISD's) encontrando os mesmos pontos de dupla saturação quando comparados com os diagramas ternários. Fez-se uma validação dos ISD comparando a posição de diferentes corridas com a energia elétrica consumida. Quanto mais próxima a corrida está da linha de saturação menor será seu consumo energético.

Palavras-chave: FEA; saturação MgO; Dupla saturação.

\section{MgO SATURATION ANALISYS OF $\mathrm{CaO}-\mathrm{SiO}_{2}-\mathrm{FeO}-\mathrm{MgO}-\mathrm{Al}_{2} \mathrm{O}_{3}$ SLAG SYSTEM}

\begin{abstract}
Electric arc furnaces (EAF) slags have multiple functions, one of them is the improvement of energy efficiency through the "slag foaming" phenomenon. It is the reason that slag studies are important to EAF's improvements. One of the main factors that determine the foamy quality and its efficiency is the presence of solid particles, so the $\mathrm{MgO}$ saturation is extremely important. Not only to guarantee the presence of solid particles, but also the chemistry compatibility, avoiding the refractory consumption. The present work aims to study the $\mathrm{MgO}$ saturation in the $\mathrm{CaO}-\mathrm{SiO}_{2}-\mathrm{FeO}-\mathrm{MgO}-\mathrm{Al}_{2} \mathrm{O}_{3}$ slag system, evaluating the influence which binary basicity, $\mathrm{FeO}$ and $\mathrm{Al}_{2} \mathrm{O}_{3}$ content have in the $\mathrm{MgO}$ saturation levels. It was carried out ternary diagrams, to see the influence that $\mathrm{Al}_{2} \mathrm{O}_{3}$ have in the liquid field and other present phases. Isothermal saturation diagrams (ISD's) were used to find the same points dual saturations that were found in the ternary diagram. To validate the ISD's some heats were plotted and them a comparison was made. The heats that were close to the $\mathrm{MgO}$ saturation line had a better energy consumption.
\end{abstract}

Keywords: EAF; MgO saturation; Dual saturation. 


\section{INTRODUÇÃO}

No forno elétrico a arco, a escória desempenha diferentes funções, desde a proteção dos refratários da radiação dos arcos, passando pela absorção de fósforo até a melhor transferência de energia para o banho.

Mas sabe-se que, para a escória desempenhar as suas funções de maneira satisfatória, a sua composição tem que ser compatível com os refratários que protegem o forno. Estes refratários, que normalmente são compostos por óxidos básicos ( $\mathrm{MgO}, \mathrm{CaO}$ etc), acabam sendo consumidos pela escória. Isso porque a escória do refino primário é rica em óxidos ácidos $\left(\mathrm{SiO}_{2}, \mathrm{MnO}, \mathrm{FeO}\right.$ etc.), necessitando que a escória de refino tenha a basicidade correta e esteja saturada em MgO.

A tabela 1 mostra um exemplo de escórias de refino primário em FEA.

Tabela 1. Principais componentes da escória de FEA e o \% da composição final [10].

\begin{tabular}{cc}
\hline Componente & Faixa de composição \\
\hline $\mathrm{CaO}$ & $25-35$ \\
\hline $\mathrm{SiO}_{2}$ & $5-15$ \\
\hline $\mathrm{FeO}$ & $25-45$ \\
\hline $\mathrm{MgO}$ & $3-8$ \\
\hline $\mathrm{CaF}_{2}$ & - \\
\hline $\mathrm{MnO}$ & $2-5$ \\
\hline $\mathrm{S}$ & - \\
\hline $\mathrm{P}_{2} \mathrm{O}_{5}$ & - \\
\hline
\end{tabular}

O excesso de $\mathrm{MgO}$ e a basicidade correta minimizam o consumo de refratário, colaboram também para o fenômeno da espumação. Fenômeno este que para ocorrer necessita de escórias com oxigênio e carbono em excesso, de acordo com as seguintes reações 1 e 2 [7].

$$
\begin{aligned}
& \underline{C}+\frac{1}{2} \mathrm{O}_{2} \rightarrow \mathrm{CO} \quad \text { Reação } 1 \\
& \mathrm{C}+(\mathrm{FeO}) \rightarrow \mathrm{CO}+\underline{\mathrm{Fe}} \quad \text { Reação } 2
\end{aligned}
$$

Com a criação das bolhas de CO geradas pelas reações 1 e 2 ocorre a expansão da escória e o recobrimento do arco. Existem diversos parâmetros que definem a qualidade da espumação da escória: alta viscosidade, baixa tensão superficial, baixa densidade [7] e a presença de partículas sólidas [9]. A saturação da escória em $\mathrm{CaO}$ e $\mathrm{MgO}$ gera partículas sólidas que servem como pontos de nucleação de bolhas, gerando bolhas pequenas e em maior quantidade [9].

O estudo da escória espumante é muito complexo, tendo variáveis como a temperatura, basicidade, composição química da escória, saturação de $\mathrm{MgO}$. Assim este trabalho tem por objetivo analisar, para a temperatura fixa de $1600^{\circ} \mathrm{C}$, $\mathrm{O}$ comportamento da saturação de $\mathrm{MgO}$ de acordo com a variação de diferentes parâmetros de composição química da escória para o sistema $\mathrm{CaO}-\mathrm{SiO}_{2}-\mathrm{MgO}-\mathrm{FeO}-$ $\mathrm{Al}_{2} \mathrm{O}_{3}$, utilizando o software de simulação termodinâmica FactSage 6.4.

\section{MATERIAIS E MÉTODOS}

Para este estudo utilizou-se o programa FactSage 6.4 para todas as determinações realizadas. Este software já foi utilizado em inúmeras pesquisas tanto de escórias de 
refino primário [1,6], quanto em escórias de refino secundário [2,3]. Neste software foi utilizado os seguintes bancos de dados para os cálculos:

- FToxid: este banco de dados é composto por duas partes, o FToxid53Soln.sda que contém as soluções oxidas avaliadas/otimizadas e o FToxid53Base.cdb contém todos os compostos sólidos e líquidos avaliados e otimizados. Neste banco de dados os óxidos principais são: $\mathrm{Al}_{2} \mathrm{O}_{3}, \mathrm{CaO}, \mathrm{FeO}, \mathrm{Fe}_{2} \mathrm{O}_{3}, \mathrm{MgO}, \mathrm{SiO}_{2}$ [11].

- FactPS: banco de dados de substâncias puras.

Para realizar a comparação e determinar o efeito que a alumina tem sobre sistema $\mathrm{CaO}-\mathrm{SiO}_{2}-\mathrm{FeO}-\mathrm{MgO}$. Utilizou-se diagramas pseudo-ternários $\mathrm{CaO}-\mathrm{SiO}_{2}-\mathrm{FeO} \mathrm{com}$ $5 \%$ de $\mathrm{MgO}$ e sem $\mathrm{Al}_{2} \mathrm{O}_{3}$ e o mesmo diagrama mas com $5 \%$ de $\mathrm{Al}_{2} \mathrm{O}_{3}$.

Outra ferramenta que pode ser utilizada para analisar a saturação de $\mathrm{MgO}$ são os diagramas de saturação isotérmicos, ISD's - Isothermal Saturation Diagrams, introduzidos por Pretorius [9]. Os ISD's são feitos considerando-se duas condições de contorno principais: a basicidade e a temperatura constantes. Na figura 1 pode-se ver um exemplo de ISD genérico.

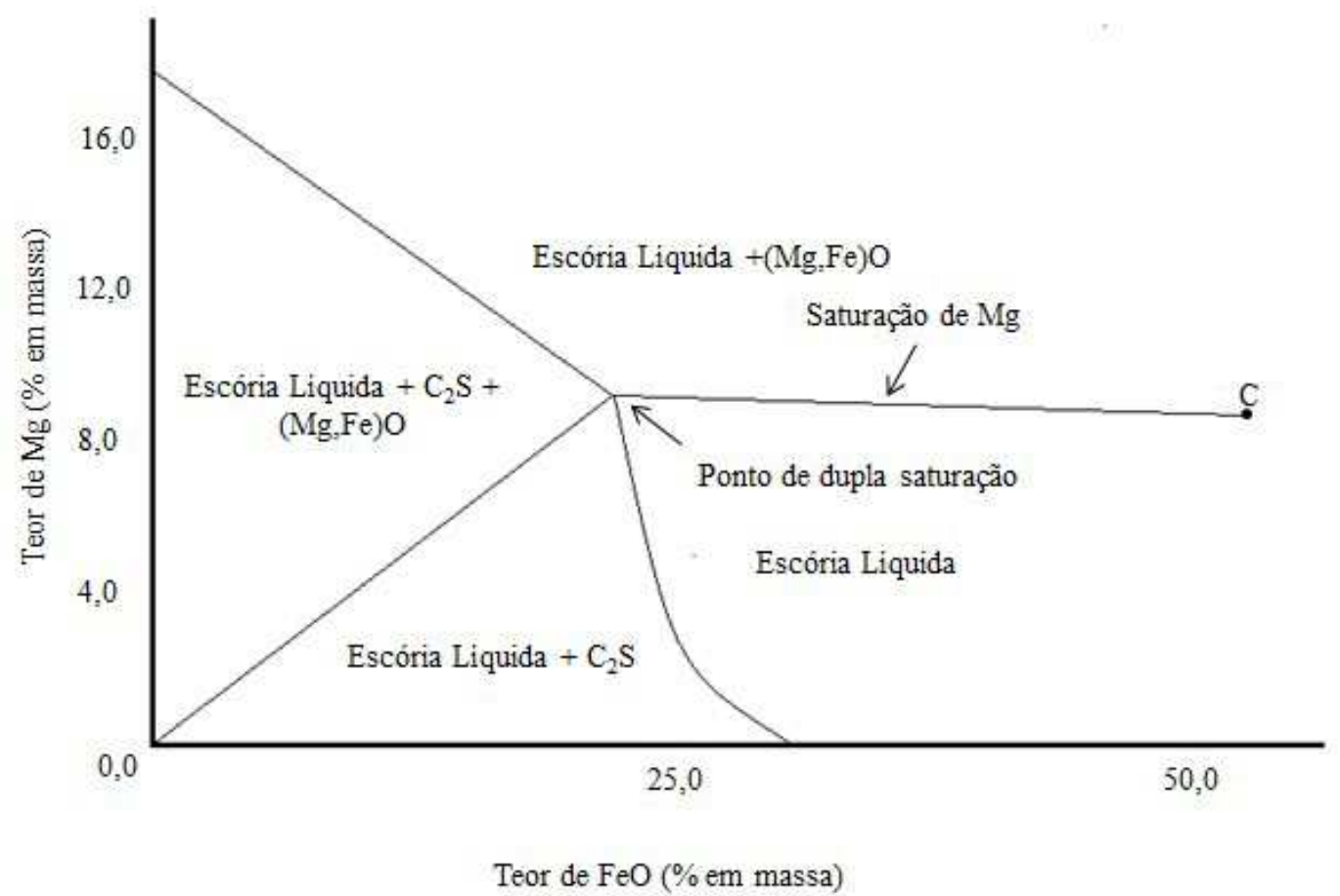

Figura 1. Diagrama de saturação isotérmico genérico [1].

A figura 1 mostra algumas das principais informações que os ISD's fornecem: teor de $\mathrm{FeO}$ e de $\mathrm{MgO}$ necessário para que ocorra a dupla saturação, o ponto $\mathrm{C}$ (que pode ter sua composição química obtida pela composição química do líquido no diagrama $\mathrm{FeO}-\mathrm{MgO}$ ) e a linha que liga o ponto de dupla saturação com o ponto $\mathrm{C}$, que seria a linha de saturação de $\mathrm{MgO}$ [4].

Sabe-se que os ISD refletem a saturação de $\mathrm{MgO}$ e que a espumação da escória é favorecida com a presença de partículas sólidas, então se a corrida está acima da linha de saturação de $\mathrm{MgO}$ mas próximo da mesma, ela terá uma espumação de melhor qualidade e um menor gasto energético.

Para a avaliação dos ISD's utilizou-se dados históricos fornecidos por uma usina de Aços Especiais. Estes dados corresponderam a corridas produzidas com um mesmo padrão de carga, ou seja, a sucata utilizada e suas quantidades foram muito semelhantes. Além disso, foram comparadas corridas que obtiveram a mesma 
composição química (basicidade e teores de $\mathrm{Al}_{2} \mathrm{O}_{3}$ ), tempos de refino próximos e temperaturas de vazamento variando no máximo $10^{\circ} \mathrm{C}$. A comparação foi feita através da posição da corrida no ISD e o gasto energético da mesma.

\section{RESULTADOS E DISCUSSÃO}

\subsection{Efeitos da Basicidade Binária e do Teor de FeO}

Sabe-se que escórias com uma maior basicidade, $\% \mathrm{CaO} / \% \mathrm{SiO}_{2}$, necessitam de um menor teor de $\mathrm{MgO}$ para saturação [1], sabe-se também que o teor de FeO tem forte influência sobre a saturação de $\mathrm{MgO}$ [5]. Esse fato pode ser evidenciado através da figura 2, que mostra o teor de $\mathrm{MgO}$ de saturação pelo teor de $\mathrm{FeO}$, nos quais se variou a basicidade binária, de 1,5 até 3,0.

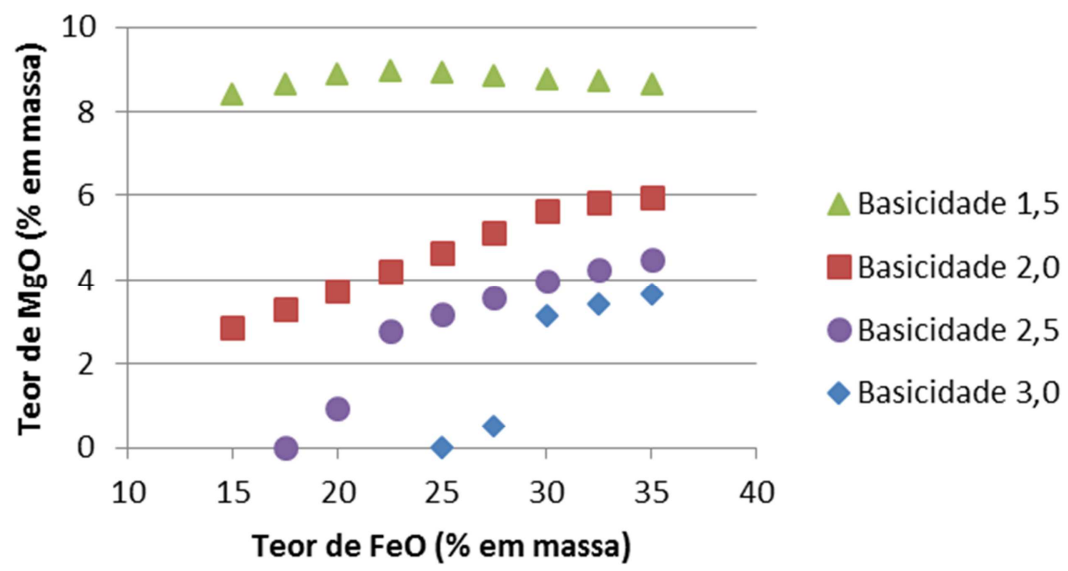

Figura 2. Efeito da basicidade no teor de saturação de $\mathrm{MgO}$, para diferentes teores de $\mathrm{FeO}$ a $1600^{\circ} \mathrm{C}$.

Pela figura 2 vê-se que para diferentes basicidades ocorrem diferentes comportamentos. Para basicidades baixas, no caso da basicidade igual a 1,5, vê-se que a saturação de $\mathrm{MgO}$ teve uma pequena variação com a mudança do teor de FeO. Para basicidades acima de 2,0 o comportamento é diferente, a saturação de $\mathrm{MgO}$ aumenta com o aumento do teor de FeO. Aumentando-se ainda mais a basicidade a escória está saturada em $\mathrm{MgO}$ até teores de $\mathrm{FeO}$ de aproximadamente $25 \%$, após esse valor o $\mathrm{MgO}$ não está saturado seguindo o mesmo comportamento dos casos anteriores. Estes três últimos casos demonstram o efeito fluxante que 0 $\mathrm{FeO}$ tem nas escórias de refino primário. Também pode-se notar que para um mesmo teor de $\mathrm{FeO}$, o MgO de saturação diminui com o aumento da basicidade.

\subsection{Efeito do Teor de $\mathrm{Al}_{2} \mathrm{O}_{3}$}

\subsubsection{Diagramas ternários}

Existe ainda outro parâmetro que influência na saturação de $\mathrm{MgO}$, que seria o teor de $\mathrm{Al}_{2} \mathrm{O}_{3}$. Pretorius em seu estudo [9] utilizou a $\mathrm{B}_{3}=\mathrm{CaO} /\left(\mathrm{SiO}_{2}+\mathrm{Al}_{2} \mathrm{O}_{3}\right)$ para realizar esta análise, substituindo uma certa quantidade de sílica por alumina, para manter a basicidade constante, pois compara uma escória sem alumina, resultando em uma basicidade binária, com uma escória com alumina, resultando em uma basicidade ternária. Para se estudar o efeito da alumina, neste trabalho foram feitos diagramas ternários $\mathrm{CaO}-\mathrm{SiO}_{2}-\mathrm{FeO}$, fixando-se os teores de $\mathrm{Al}_{2} \mathrm{O}_{3}$ e de $\mathrm{MgO}$, para que pudesse ser feita a comparação. Nas Figuras 3 e 4 estão os diagramas pseudo-ternários com $5 \%$ de $\mathrm{MgO}$ com e sem $\mathrm{Al}_{2} \mathrm{O}_{3}$. 


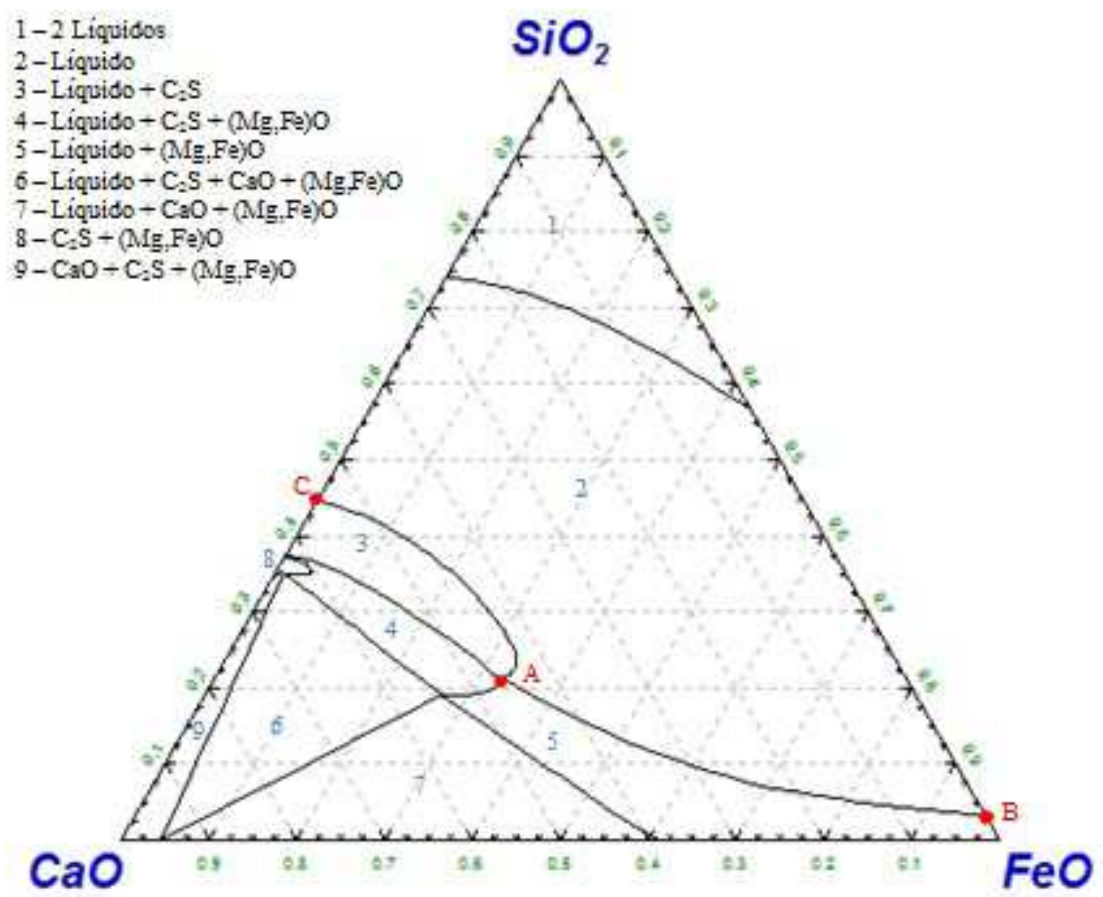

Figura 3. Diagrama pseudo-ternário $\mathrm{CaO}-\mathrm{SiO}_{2}-\mathrm{FeO}$, com $5 \%$ de $\mathrm{MgO}$ e $\mathrm{T}=1600^{\circ} \mathrm{C}$.

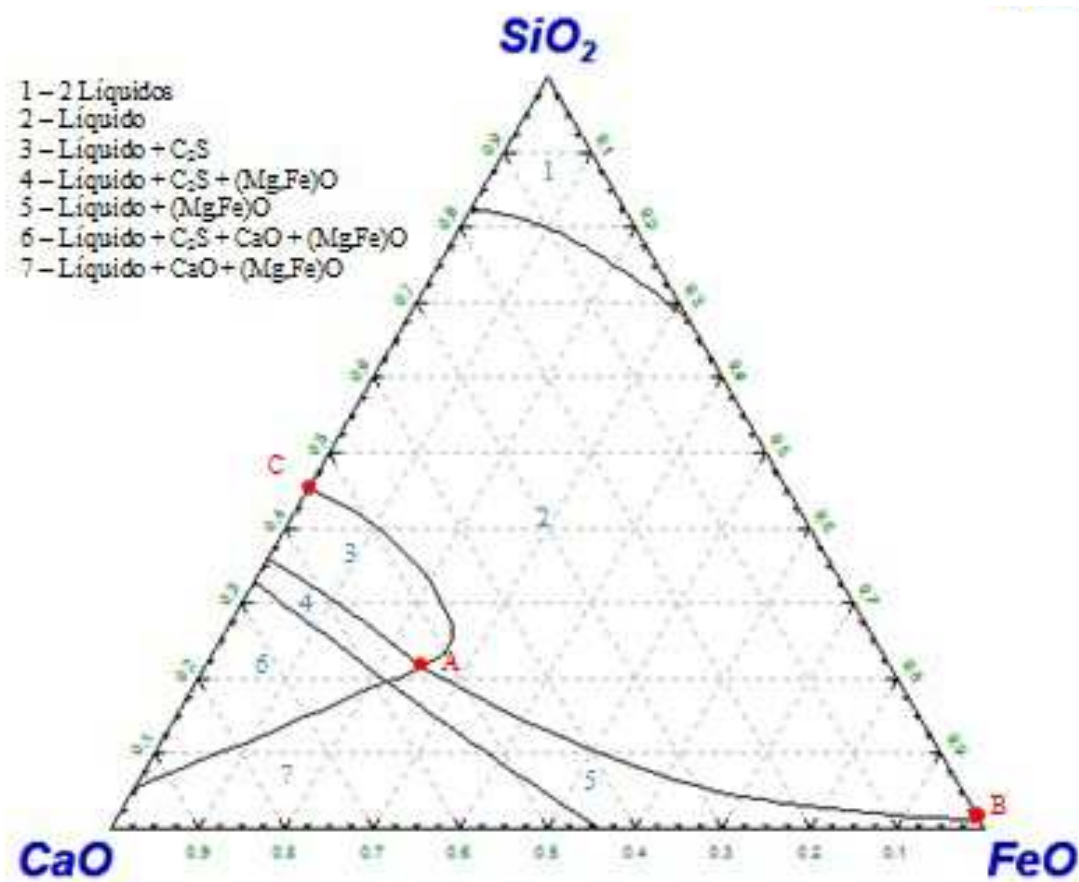

Figura 4. Diagrama pseudo-ternário $\mathrm{CaO}-\mathrm{SiO}_{2}-\mathrm{FeO}$ com $5 \%$ de $\mathrm{Al}_{2} \mathrm{O}_{3}$ e $5 \%$ de $\mathrm{MgO}$ e $\mathrm{T}=1600^{\circ} \mathrm{C}$.

Os campos de maior interesse neste trabalho são os campos 2, 3 e 5, esses campos são compostos por: líquido, líquido $+\mathrm{C}_{2} \mathrm{~S}$ e líquido $+(\mathrm{Mg}, \mathrm{Fe}) \mathrm{O}$, respectivamente. Ou seja, no campo 3 ocorre a precipitação do $\mathrm{CaO}$, na forma de $\mathrm{C}_{2} \mathrm{~S}\left(2 \mathrm{CaO} . \mathrm{SiO}_{2}\right)$, no campo 5 ocorre a saturação de $\mathrm{MgO}$, na forma de ( $\mathrm{Mg}, \mathrm{Fe}) \mathrm{O}$ (magnésio-wustita). Para que ocorra uma melhor visualização das diferenças entre os dois diagramas, foi feita uma sobreposição mostrada na figura 5. 


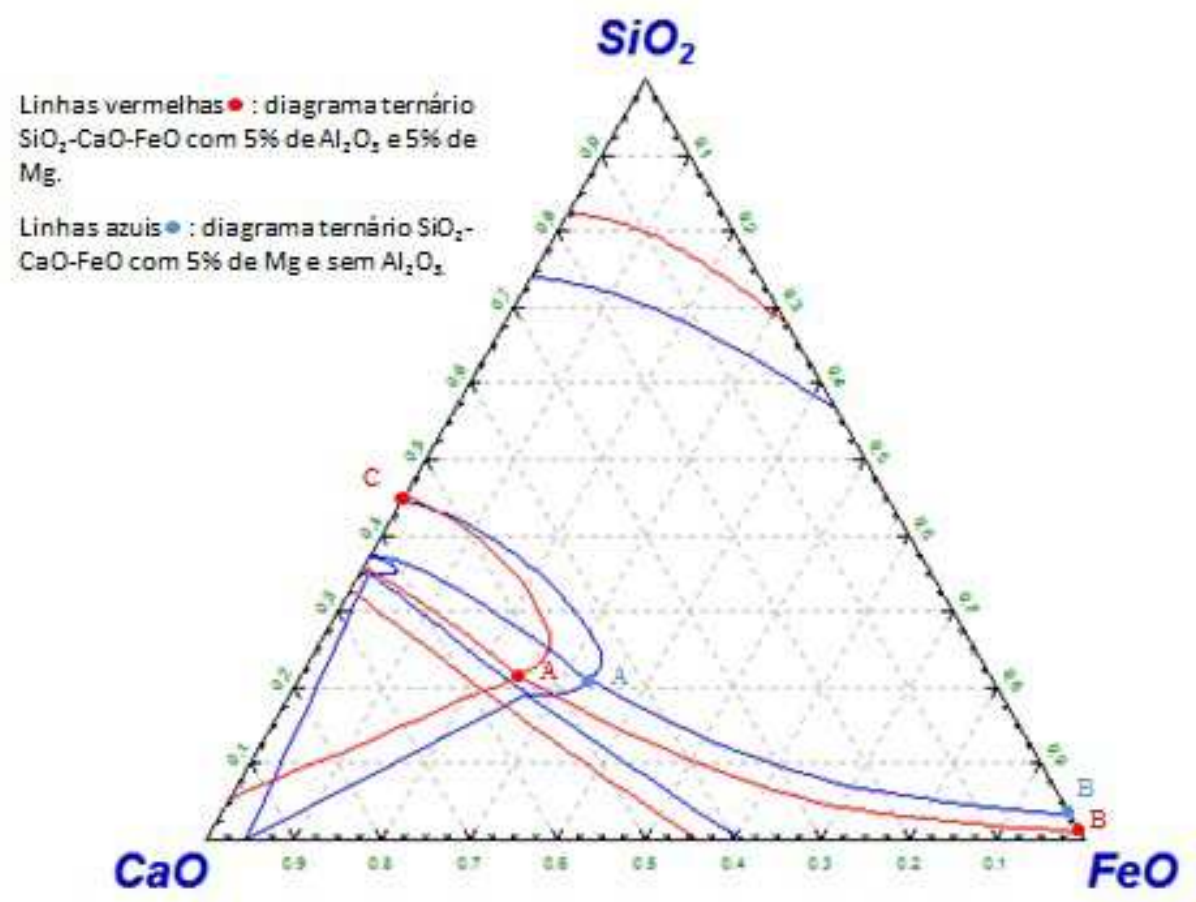

Figura 5. Sobreposição dos diagramas pseudo-ternários das figuras 3 e $4, T=1600^{\circ} \mathrm{C}$.

$\mathrm{Na}$ figura 5 notam-se as diferenças entre os diagramas ternários. Com a presença de $\mathrm{Al}_{2} \mathrm{O}_{3}$ ocorre a expansão do campo de escória líquida (campo 2), visto através da mudança nas linhas de saturação do $\mathrm{C}_{2} \mathrm{~S}$ e da magnésio-wustita, linhas A-C e A-B respectivamente. Nota-se também a supressão do campo que é composto por $\mathrm{C}_{2} \mathrm{~S}+$ magnésio-wustita, campo 8 da figura 3, assim como a supressão do campo formado por $\mathrm{CaO}+\mathrm{C}_{2} \mathrm{~S}+$ Magnésio-wustita sólidos, campo 9 da figura 3 .

Outra diferença importante ocorre para o ponto A, segundo Paulino [8] é o ponto de dupla saturação. Ou seja, é o ponto de intersecção dos campos 2, 3 e 5 das figuras 3 e 4, neste ponto ocorre a saturação tanto do $\mathrm{CaO}$, na forma de $\mathrm{C}_{2} \mathrm{~S}$ quanto a saturação de $\mathrm{MgO}$, na forma de magnésio-wustita.

Houve uma grande mudança no ponto $A$ com a mudança no teor de $\mathrm{Al}_{2} \mathrm{O}_{3}$, na figura 3 (linhas azuis da figura 5), o ponto $\mathrm{A}$ tem a seguinte composição química: $\mathrm{SiO}_{2}=$ $20,21 \%, \mathrm{CaO}=43,60 \%, \mathrm{FeO}=30,59 \%$ e $\mathrm{MgO}=5 \%$; mas na figura 4 (linhas vermelhas da figura 5) o ponto $A$ está com a seguinte composição química: $\mathrm{SiO}_{2}=$ $19,40 \%, \mathrm{CaO}=48,46 \%, \mathrm{FeO}=22,13 \%, \mathrm{MgO}=5 \%$ e $\mathrm{Al}_{2} \mathrm{O}_{3}=5 \%$. Mostrando a mudança que a presença de $5 \%$ de $\mathrm{Al}_{2} \mathrm{O}_{3}$ causa, sendo necessário uma maior basicidade binária $\left(\% \mathrm{CaO} / \% \mathrm{SiO}_{2}\right)$, aumentando de 2,15 para 2,5 , assim como um menor teor de $\mathrm{FeO}$, diminuindo de $30,59 \%$ para $22,13 \%$, para que ocorra a dupla saturação, evidenciando o efeito fluxante da alumina.

\subsubsection{Diagramas de saturação isotérmicos}

Como os ISD's são feitos utilizando-se basicidade e temperatura constantes, foram feitos ISD's utilizando-se as composições químicas encontradas nos pontos A's dos diagramas pseudo-ternários das figuras 3 e 4, ou seja, utilizaram-se as mesmas basicidades encontradas, o mesmo teor de $\mathrm{Al}_{2} \mathrm{O}_{3}$ e temperatura de $1600^{\circ} \mathrm{C}$. Comparou-se os teores de $\mathrm{FeO}$ e de $\mathrm{MgO}$ encontrados nos diagramas pseudoternários para o ponto de dupla saturação (ponto A), com os valores encontrados nos ISD's das figuras 6 e 7. 


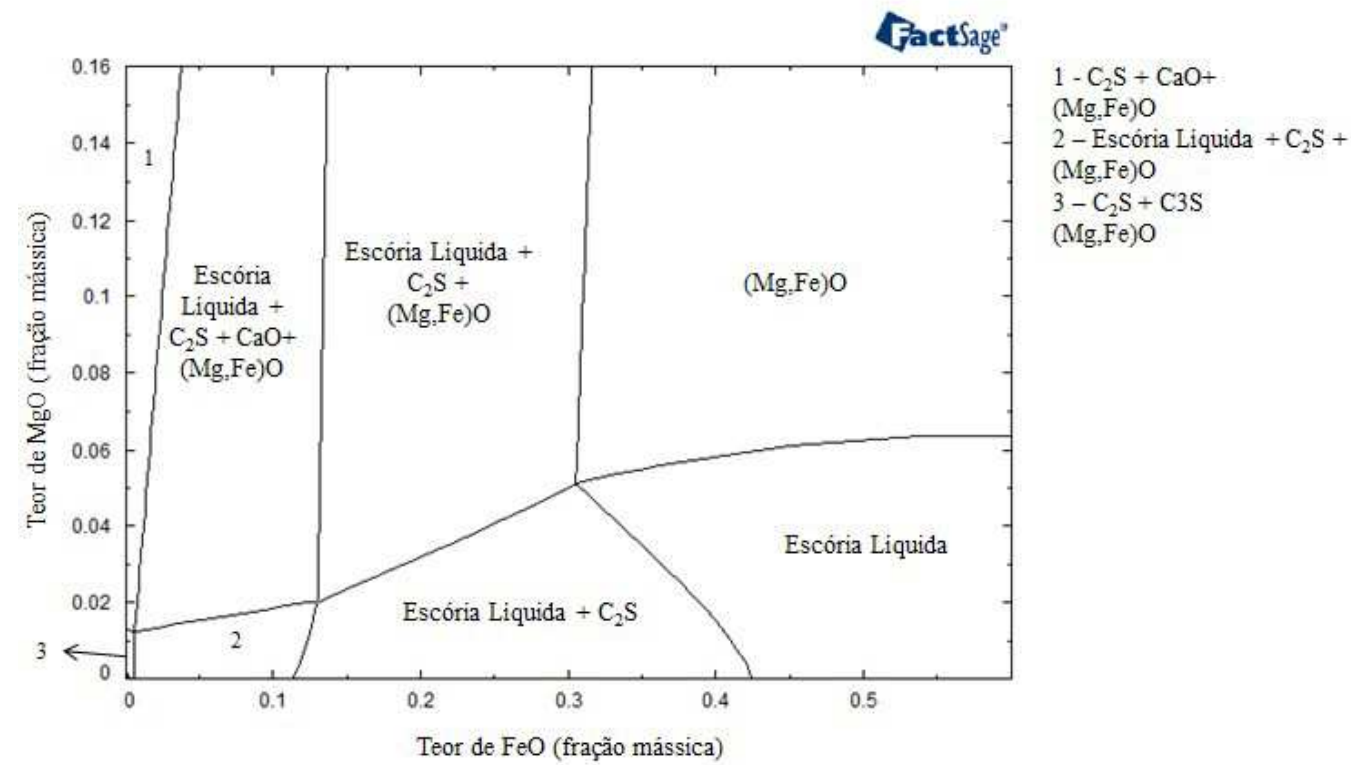

Figura 6. Diagrama de saturação isotérmico $B_{2}=2,15$ e $T=1600^{\circ} \mathrm{C}$.

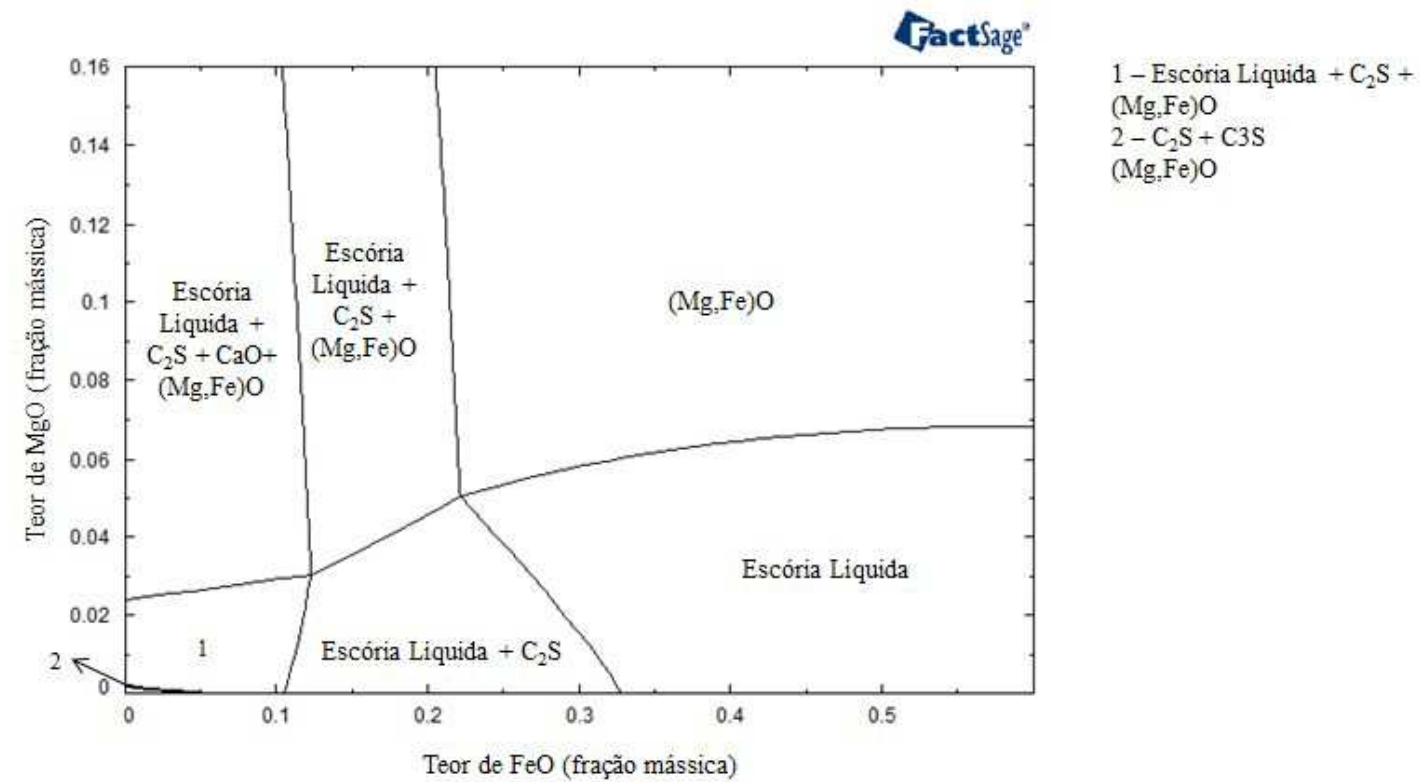

Figura 7. Diagrama de saturação isotérmico $B_{2}=2,5$ e $T=1600^{\circ} \mathrm{C}$.

O ISD que está na figura 6 , foi feito para a basicidade 2,15 e sem a presença de $\mathrm{Al}_{2} \mathrm{O}_{3}$, neste gráfico o ponto de dupla saturação está com a seguinte composição química: teor de $\mathrm{FeO}=30,5 \%$ e o teor de $\mathrm{MgO}=5,07 \%$. Esses valores os mesmos encontrados utilizando-se o diagrama ternário (figura 3). Na figura 7, o ISD foi construído com a basicidade igual a 2,5, neste gráfico o ponto de dupla saturação foi encontrado em $\mathrm{FeO}=22,25 \%$ e $\mathrm{MgO}=5,01 \%$, sendo estes dados iguais aos encontrados para o ponto A da figura 4. Isso mostra que os ISD podem ser utilizados para prever o comportamento de escórias com composição químicas complexas, com a presença de $\mathrm{Al}_{2} \mathrm{O}_{3}$, por exemplo.

Para avaliar o efeito que $0 \mathrm{Al}_{2} \mathrm{O}_{3}$ tem sobre a saturação de $\mathrm{MgO}$, utilizou-se os ISD's. As diferenças podem ser vistas na figura 8 , foram feitas sobreposições dos gráficos para facilitar a visualização para as basicidades binárias de 1,5, 2,0, 2,5 e 3. 

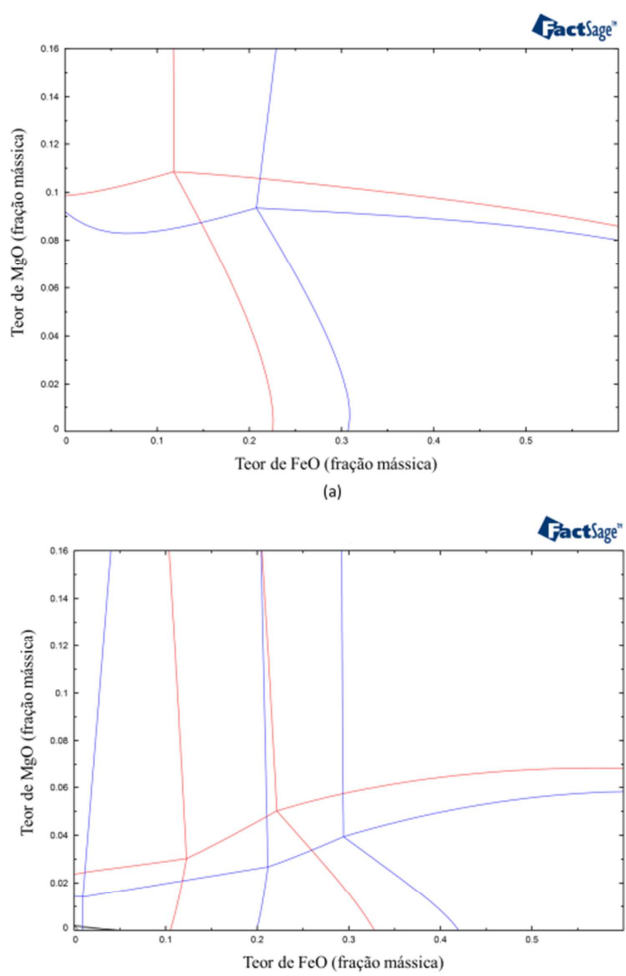
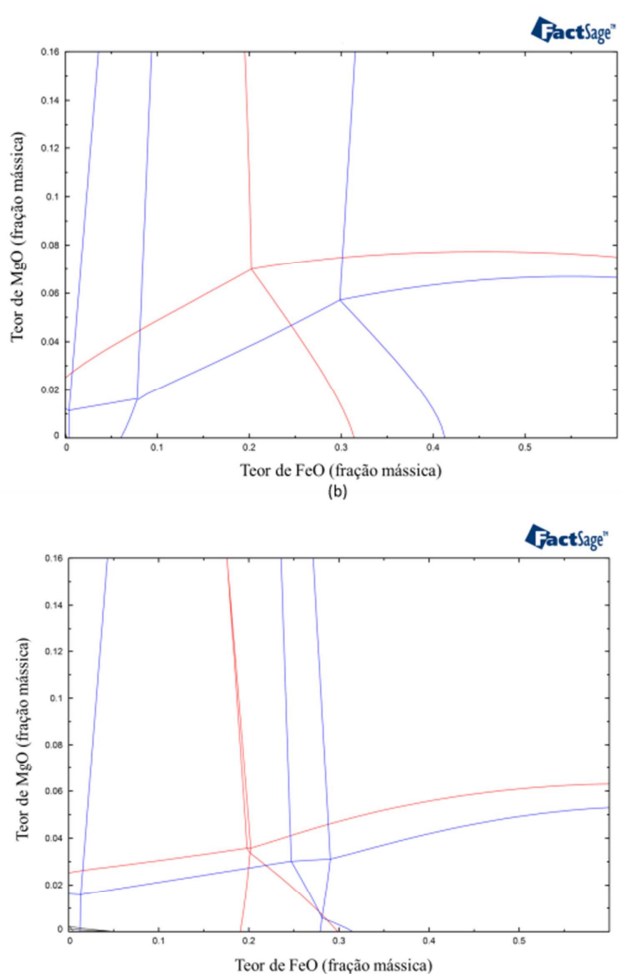

(d)

Figura 8. Diagramas de saturação isotérmicos para diferentes basicidades binárias, linhas azuis diagramas feitos sem a presença de $\mathrm{Al}_{2} \mathrm{O}_{3}$, linhas vermelhas diagramas feitos considerando a presença de $5 \%$ de $\mathrm{Al}_{2} \mathrm{O}_{3}$ e $\mathrm{T}=1600^{\circ} \mathrm{C}$. (a) basicidade binária 1,5; (b) basicidade binária 2,0; (c) basicidade binária 2,5; (d) basicidade binária 3,0.

Para as quatro basicidades vê-se que a alumina tem os mesmos efeitos nos ISD's. Ocorre o aumento do $\mathrm{MgO}$ necessário para a saturação, ocorre também a diminuição do $\mathrm{FeO}$ necessário para a dupla saturação, como no caso do ISD com basicidade 2,0, o FeO necessário para a dupla saturação caiu de $30 \%$ para $20 \%$, com estes dois efeitos somados ocorre o mesmo que foi visto para os diagramas pseudo-ternários, há um aumento no campo líquido.

\subsubsection{Testes com dados industriais}

Com os dados industriais recebidos, plotou-se nos ISD's das figuras 9, 10 e 11, as corridas com o mesmo teor de alumina e a mesma basicidade que foram utilizadas para a confecção destes ISD's.

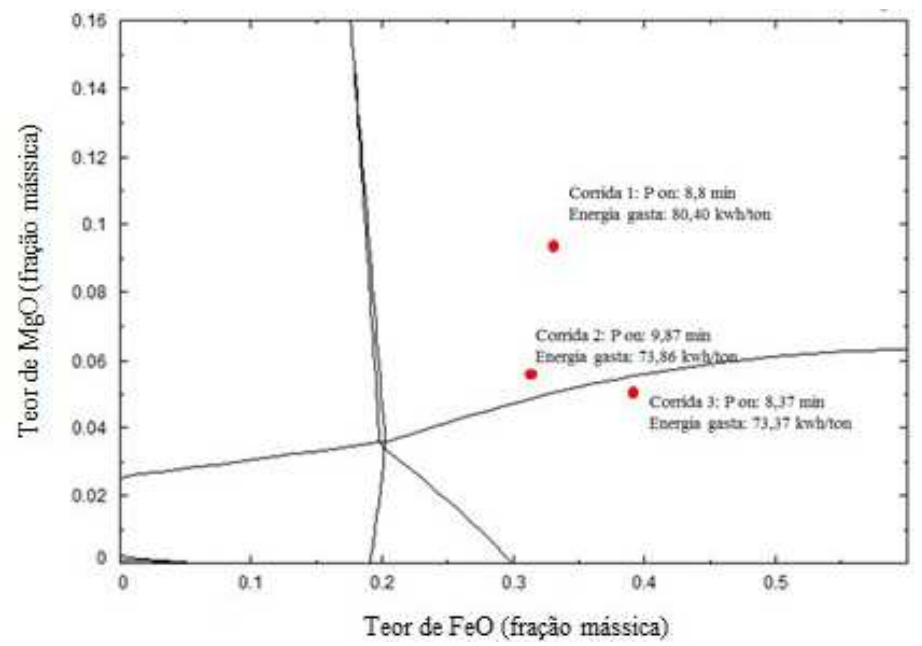

Figura 9. Diagrama de saturação isotérmico com $5 \%$ de $\mathrm{Al}_{2} \mathrm{O}_{3}$ e $\mathrm{B}_{2}=3,0$ e $\mathrm{T}=1600^{\circ} \mathrm{C}$. 


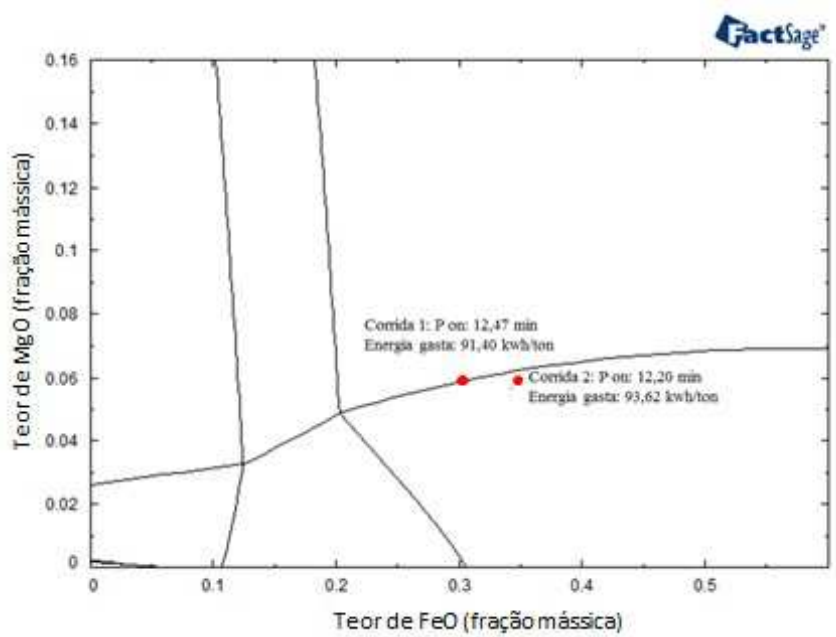

Figura 10. Diagrama de saturação isotérmico com $6 \%$ de $\mathrm{Al}_{2} \mathrm{O}_{3}$ e $\mathrm{B}_{2}=2,6$ e $\mathrm{T}=1600^{\circ} \mathrm{C}$.

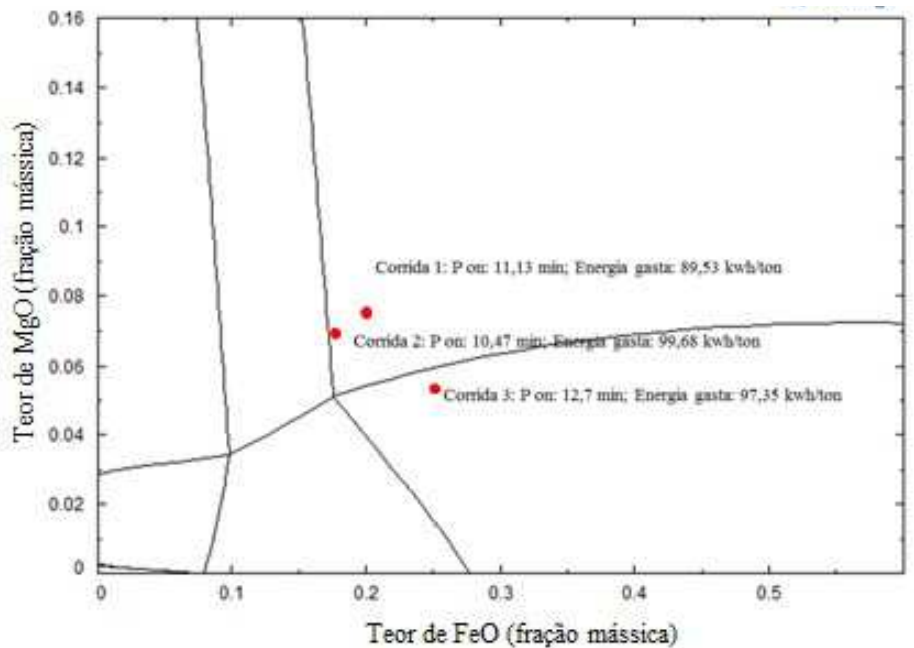

Figura 21. Diagrama de saturação isotérmico com $7,5 \%$ de $\mathrm{Al}_{2} \mathrm{O}_{3}$ e $\mathrm{B}_{2}=2,6$ e $\mathrm{T}=1600^{\circ} \mathrm{C}$.

Para as figuras 9 a 11, nota-se o mesmo comportamento das corridas, quanto mais próximo da linha de saturação de $\mathrm{MgO}$, menor foi o consumo de energia elétrica. Corridas que estão logo acima da linha (corrida 2 da figura 9), obtiveram um gasto energético menor ou igual se comparadas com corridas que estão logo abaixo da linha de saturação (corrida 3 da figura 9) mesmo com a diferença de 1 minuto no tempo de power on.

\section{CONCLUSÕES}

- O programa FactSage apresentou resultados conforme o esperado para a relação da basicidade com a saturação de $\mathrm{MgO}$, onde há uma diminuição do $\mathrm{MgO}$ necessário para saturação com o aumento da basicidade. Assim como a relação do teor de $\mathrm{FeO}$ com a saturação de $\mathrm{MgO}$, quando as basicidades foram de 2,0 ou maior houve um aumento no $\mathrm{MgO}$ de saturação. Para o caso da basicidade de 1,5 houve uma pequena variação no $\mathrm{MgO}$ de saturação de acordo com o aumento do teor de FeO.

- O efeito do $\mathrm{Al}_{2} \mathrm{O}_{3}$ foi visto através dos diagramas pseudo-ternários $\mathrm{CaO}-\mathrm{SiO}_{2}$ $\mathrm{FeO} 5 \% \mathrm{MgO}$ e $\mathrm{CaO}-\mathrm{SiO}_{2}-\mathrm{FeO} 5 \% \mathrm{MgO}$ e $5 \% \mathrm{Al}_{2} \mathrm{O}_{3}$. Notou-se uma grande diferença no campo líquido, assim como no ponto de dupla saturação, que 
teve a sua composição química modificada de $\mathrm{SiO}_{2}=20,21 \%, \mathrm{CaO}=43,60 \%$, $\mathrm{FeO}=30,59 \%$ e $\mathrm{MgO}=5 \%$; para $\mathrm{SiO}_{2}=19,40 \%, \mathrm{CaO}=48,46 \%, \mathrm{FeO}=$ $22,13 \%, \mathrm{MgO}=5 \%$ e $\mathrm{Al}_{2} \mathrm{O}_{3}=5 \%$, mostrando uma grande queda no teor de $\mathrm{FeO}$ e um aumento na basicidade, indo de 2,15 para 2,5, para que ocorra a dupla saturação.

- Os ISD's feitos para os pontos mencionados nos diagramas pseudo-ternários mostraram os mesmos pontos de dupla saturação, tendo o teor de $\mathrm{MgO}$ em $5 \%$ e o de $\mathrm{FeO}=30,5 \%$ para a basicidade 2,15 e teor de $\mathrm{MgO}=5 \%$ e de $\mathrm{FeO}=22,25 \%$ para a basicidade 2,5 .

- A comparação dos ISD's com e sem alumina mostrou o efeito fluxante da alumina, sendo necessário um maior teor de $\mathrm{MgO}$ para a saturação e um menor teor de FeO para que ocorra a dupla saturação.

- Os ISD's podem ser utilizados para análise da saturação de MgO, pois para corridas com power on de refino próximos, quanto mais perto da linha de saturação, menor foi o gasto energético.

\section{Agradecimentos}

Rodolfo Almeida agradece aos professores Wagner Bielefeldt e Antônio Vilela pela orientação e apoio, como aos colegas do LASID e ao CNPq pela bolsa de estudos.

\section{REFERÊNCIAS}

1 Bennet, J, Kwong, KS. Thermodynamic Studies of MgO Saturated EAF Slags, Ironmaking and Steelmaking, Vol 37, No 7, pp.529 - 535, 2010.

2 Bielefeldt, WV. Evaluation of the Slag System CaO-MgO- $\mathrm{Al}_{2} \mathrm{O}_{3}-\mathrm{SiO}_{2}, 44_{\text {th }}$ Steelmaking Seminar, 2013.

3 Bielefeldt, WV. Thermodynamic Evaluation of the Slag System CaO-MgO-SiO${ }_{2}-\mathrm{Al}_{2} \mathrm{O}_{3}$, $69^{\text {th }}$ Congresso Anual da ABM, 2013.

4 Kwong, KS, Bennet, J. Achieving MgO Saturated Foamy Slags in the EAF, Electric Furnace Conference Proceedings, 2001.

$5 \mathrm{Kim}, \mathrm{YJ}$, Min DJ. Effect of $\mathrm{FeO}$ and $\mathrm{Al}_{2} \mathrm{O}_{3}$ on the $\mathrm{MgO}$ Solubility in $\mathrm{CaO}-\mathrm{SiO}_{2}-\mathrm{FeO}-\mathrm{Al}_{2} \mathrm{O}_{3}-$ MgO Slag System at 1823K. Steel Research International, Vol. 83, No 9, 2012.

6 Luz AP, Thermodynamic simulation and Isothermal Solubility Diagram as Tools for Slag Foaming Control, Ceramic International 37, 2011.

7 Morales, RD. The Slag Foaming Practice in EAF and Its Influence on the Steelmaking Shop Productivity, ISIJ International, Vol 35, No. 9, pp. 1054-1062, 1995.

8 Paulino, MAS. Estudo da Escória Espumante em Forno Elétrico a Arco, Congresso Anual da ABM, São Paulo, 2014.

9 Pretorius, EB. Foamy Slag Fundamentals and their Practical Application to Electric Furnace Steelmaking, $55^{\circ}$ Congresso Anual da ABM, 2000.

10 Turkdogan, ET, Fruehan, RJ. The Making, Shaping and Treating of Steel. Steelmaking and Refining - the AISE Steel Fundation, vol 2, 1998

11 FactSage: [http://www.crct.polymtl.ca/fact/documentation/], acessado em 03/03/2015 